\title{
Effects of Optical Artifacts in a Laser-Based Spacecraft Navigation Sensor
}

\author{
Jerry E. LeCroy ${ }^{1}$ \\ Boeing Company, Huntsville, Alabama 35824 \\ Richard T. Howard ${ }^{2}$ \\ NASA - Marshall Space Flight Center, Huntsville, Alabama 35812 \\ and \\ Dean S. Hallmark ${ }^{3}$ \\ Boeing Company, Huntsville, AL 35824
}

\begin{abstract}
Testing of the Advanced Video Guidance Sensor (AVGS) used for proximity operations navigation on the Orbital Express ASTRO spacecraft exposed several unanticipated imaging system artifacts and aberrations that required correction to meet critical navigation performance requirements. Mitigation actions are described for a number of system error sources, including lens aberration, optical train misalignment, laser speckle, target image defects, and detector nonlinearity/noise characteristics. Sensor test requirements and protocols are described, along with a summary of test results from sensor confidence tests and system performance testing.
\end{abstract}

\section{Nomenclature}

$\begin{array}{ll}\text { ASTRO } & =\text { Autonomous servicing satellite } \\ \text { AVGS } & =\text { Advanced Video Guidance Sensor } \\ A z & =\text { azimuth angle } \\ E l & =\text { elevation angle } \\ \text { ILOAD } & =\text { initialization load } \\ I T C & =\text { ILOAD tilt constant } \\ \text { LOS } & =\text { line of sight } \\ \text { NEXTSat } & =\text { Next generation serviceable satellite }\end{array}$

\section{AVGS System Description and Application}

he Advanced Video Guidance sensor system combines an imaging sensor, integrated laser sources, and narrow-band-filtered retroreflective targets with sophisticated signal processing and optical correlation to develop six-degree-of-freedom estimates of relative state between two spacecraft engaged in proximity operations.

\section{A. Sensor/Target Description}

Figure 1 depicts the AVGS sensor optics, including lens, one megapixel complementary metal oxide semiconductor (CMOS) imager, diode laser sources driving fiber optic emitters, and retroreflector targets. The laser sources emit at two discrete wavelengths, $808 \mathrm{~nm}$ (background) and $845 \mathrm{~nm}$ (foreground). Laser power is coupled into a fiber optic combiner that is mounted in a turning mirror coaxial with the imaging system. The AVGS unit also contains image processing and communication electronics.

\footnotetext{
${ }^{1}$ Senior Engineer, Integrated Defense Systems, 950 Explorer Blvd, MC JV-03

${ }^{2}$ Tearn Leader, Advanced Vehicle Sensors, EV21

${ }^{3}$ Senior Engineer, Integrated Defense Systems, 950 Explorer Blvd, MC JV-03, AIAA Regular Member
} 


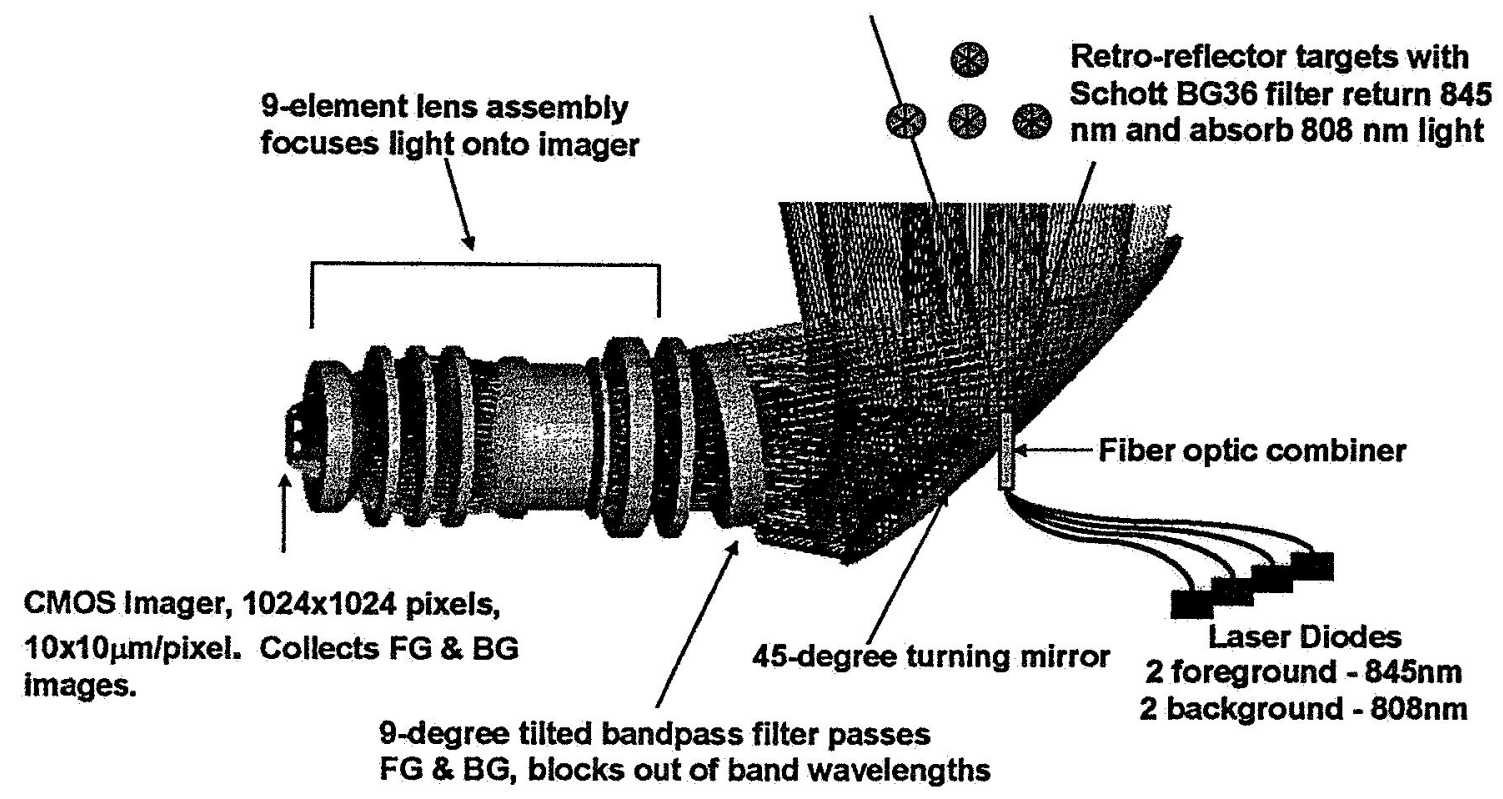

Figure 1. Advanced Video Guidance Sensor Optical Train

Light from the laser sources illuminates system-specific target clusters" within the sensor field of view (FOV). Each target retroreflector contains a precision solid corner cube, a colored glass filter to reject the background laser source and pass foreground wavelengths, and a weak plano-concave lens. AVGS operates by performing a subtraction of images with background illumination from images with foreground illumination. Image subtraction provides excellent differentiation of AVGS targets from the varied optical background presented by spacecraft in orbital lighting conditions.

AVGS software and firmware perform several tasks in each measurement frame to produce navigation solutions:

1) Based on range estimates, sets integration time and drives lasers at appropriate power to create usable and unsaturated target images

2) Collects foreground and background images

3) Subtracts a table-defined threshold value and the background image from the foreground image, to produce a derivative image with target spots well differentiated

4) Evaluates spots on subtracted image for size, aspect ratio, and image saturation to recognize potentially valid target spots

5) Evaluates candidate spots identified as potentially valid for valid target patterns and selects target sets that represent valid target cluster geometry

6) Based on knowledge of target cluster geometry, performs a perspective inversion operation to calculate range and position/attitude quaternions defining relative navigation state between spacecraft

7) Formats tracking data along with housekeeping and system health data and transmits combined data stream to the spacecraft guidance and control computer

The imaging and image processing tasks are performed at $10 \mathrm{~Hz}$, with solutions averaged and reported to the navigation system at $5 \mathrm{~Hz}$.

\section{B. Orbital Express Application}

The Orbital Express Demonstration System comprises two independent spacecraft: one prototype satellite servicing vehicle (ASTRO) and one prototype of an on-orbit-serviceable spacecraft (NEXTSat). The ASTRO 
vehicle will perform approach, docking, and separation maneuvers under multiple lighting conditions and approach trajectories. While docked the ASTRO performs propellant transfer and hardware resupply operations with the NEXTSat. On-orbit-replaceable units (ORUs) are transferred using a robotic arm.

\section{Mission Performance Requirements Derived for AVGS}

The principal requirement for the ASTRO navigation sensors, including AVGS, is to provide highly accurate and reliable relative state navigation solutions to vehicle guidance. During proximity operations the guidance system updates at a five $\mathrm{Hz}$ rate, which defines the sensor output rate requirement. AVGS operates during the terminal approach segments of proximity operations at ranges between 120 meters and fully docked (1.1 meters in sensor coordinates).

Accurate navigation requirements derive from two operational imperatives: minimizing propellant consumption and collision avoidance during approach and docking. Propellant consumption during the "forced motion" terminal approach segment is directly related to navigation measurement accuracy, so even at extended ranges the navigation errors need to be minimized to prevent excessive propellant usage. Accurate positioning in the docking "box" is required for successful capture and docking maneuvers. Small errors less than $5 \mathrm{~cm}$ in the navigation sensor could cause positioning errors that would prevent successful capture. Larger errors could even lead to spacecraft collision.

NEXTSat and ASTRO, as demonstration system components, are smaller than most candidates for operational serviceable/servicing spacecraft, and the docking mechanisms are scaled to the spacecraft size. As a result, the dimensions of the docking box, shown in Figure 2, are limited to approximately $+/-5.0$ centimeters in range and $+/-$ $4.5 \mathrm{~cm}$ along the spacecraft lateral ( $\mathrm{y}$ and $\mathrm{z}$ ) axes. These critical parameters define the allowable error budget for the combined errors of navigation sensors, hardware placement, state propagation estimator, guidance, and control. The navigation sensors, including AVGS, are allocated approximately one third of the available error budget. The baseline error allocation for AVGS at ranges closer than three meters is $15 \mathrm{~mm}$.

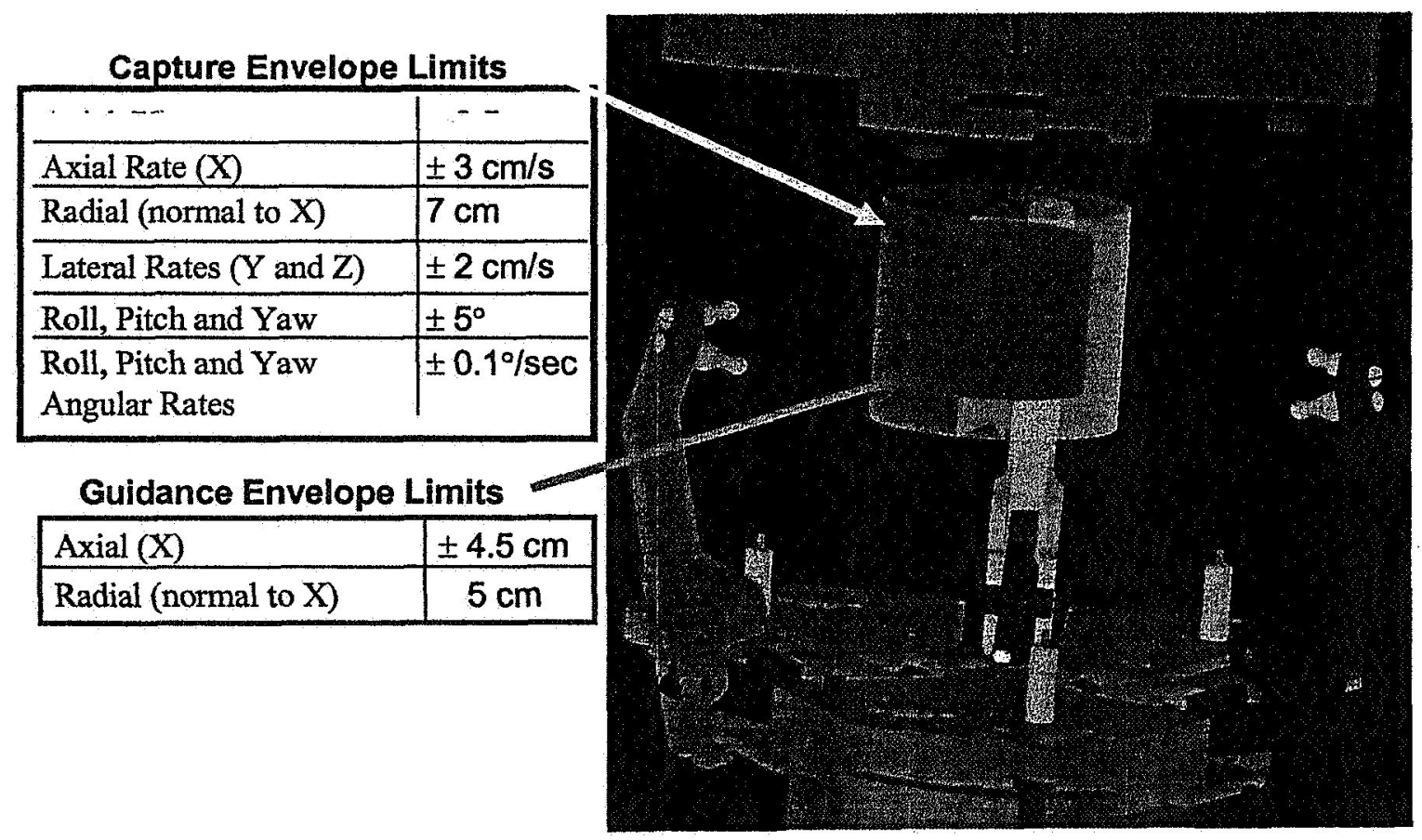

Figure 2. ASTRO vehicle docking box dimensions with allowable errors for vehicle proximity operations navigation sensors.

Since vehicle collision avoidance is critical, it is crucial that the sensor tracking performance is uninterrupted as ASTRO approaches the client vehicle. If the AVGS were to drop track for a two second period, the ASTRO guidance system will execute a collision avoidance/abort maneuver. An un-planned collision avoidance/abort maneuver would cause a delay in mission timelines and add substantial propellant expenditures. The sensitivity of 
the combined vehicle system to sensor accuracy and reliability drive the AVGS sensor performance requirements detailed in Error! Reference source not found.

Table 1. AVGS Technical Performance Requirements

\begin{tabular}{|l|c|}
\hline \multicolumn{1}{|c|}{ Technical Performance Detail } & Performance Requirement \\
\hline Acquisition and tracking range & 200 meters to dock \\
\hline Range accuracy within docking box & $+/-15 \mathrm{~mm}$ \\
\hline $\begin{array}{l}\text { Lateral alignment measurement accuracy within } \\
\text { docking box }\end{array}$ & $+/-15 \mathrm{~mm}$ \\
\hline $\begin{array}{l}\text { Relative attitude measurement accuracy within } \\
\text { docking box }\end{array}$ & $+/-0.4$ degrees, roll, pitch, and yaw \\
\hline Navigation data reporting rate/latency & $5 \mathrm{~Hz} /<175$ milliseconds \\
\hline
\end{tabular}

\section{Discussion of Optical Sensor Error Sources}

Imaging sensors used for navigation inevitably include a number of potential error sources. Error sources that would be negligible in conventional imaging applications will create unacceptable measurement bias or noise when the imager is used as a navigation source. Some error sources are peculiar to the AVGS application. For instance, star trackers (used for attitude determination) image stars that are inherently point sources. In contrast, because the AVGS target ranges vary so widely as the spacecraft executes approach trajectories, the target images vary between near-point sources and large extended sources. The large variation in the target image characteristics presents multiple opportunities for errors to develop. All of the potential errors must be accommodated by the sensor's image processing software.

\section{A. Optical System Alignment}

The most obvious error sources are in optical system alignment. Simple boresight misalignment relative to the sensor mounting interface to the spacecraft could be accommodated in guidance software. If the sensor focal plane is not precisely aligned with the lens optical axis, boresight and distortion errors emerge. Simple compensation for fixed bias in boresight alignment can be straightforward. If the boresight offset places the focal plane off-axis, that will substantially complicate any corrections required to account for lens spherical distortion.

\section{B. Alignment Effects on Solution Accuracy}

Basic alignment errors are accommodated in an AVGS initialization data table. If not addressed, these biases could easily exceed the allowable error limits for azimuth, elevation, and roll alignment errors.

\section{Alignment Accuracy}

Optical alignment is performed during sensor final assembly and checked after acceptance vibration testing of each axis using the sensor's imager. Post-assembly verification that the image plane is orthogonal to the boresight axis is not easily accomplished. Resulting optical effects may be seen as distortion of image shapes as the image is formed further off-axis.

\section{Alignment Stability}

Alignment stability must be maintained through the launch environment and during operations. The sensor alignment offsets must remain essentially identical to those measured during ground testing, even following exposure to launch dynamics and during varying on-orbit thermal conditions. To remain stable throughout the operational scenarios, the design must control temperature variations in the imaging system, and resultant asymmetric thermal deflections. Some alignment variation is unavoidable on any optical system which is not isothermalized.

The AVGS contains two active thermal control systems to manage focal plane and laser temperatures. The optical bench is mounted semi-kinematically and partially isolated thermally, but some thermal displacement of images remains. Figure 3 depicts the long term variation in target centroids at a single target range. The periodicity of target azimuth and elevation variation appears to match the duty cycle of the thermo-electric devices used to 
stabilize operating temperatures for the focal plane and lasers. Some improvement in sensor stability could be obtained if the thermal control system exerted proportional control, rather than using a simple bang-bang thermostat.

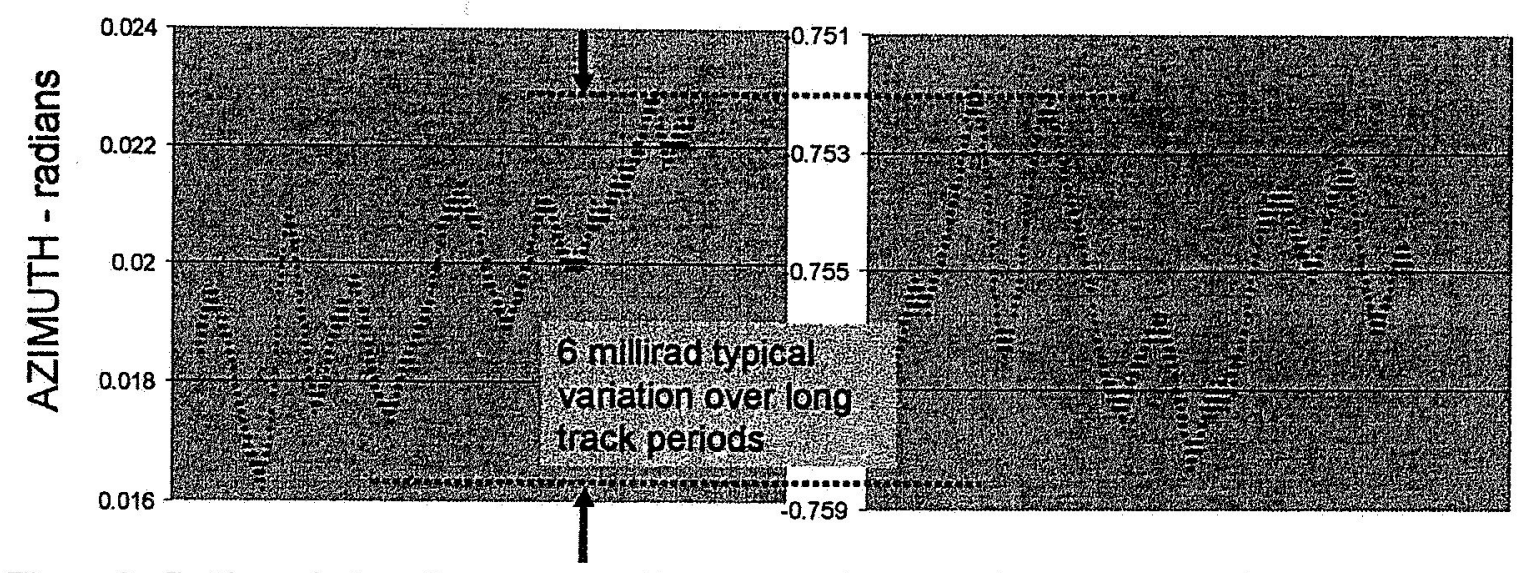

Figure 3. Cyclic variation of target centroids over 20 minute tracking periods attributed to cycling of laser and imager thermal control systems

\section{E. Optical Distortion/Aberrations}

There are several possible sources of optical distortion in the AVGS: the lens, the mirror, and the position of the CMOS imager with respect to the lens. Each of these sources results in one or more types of distortion. In addition, the corner-cube retro-reflectors induce error into the system.

\section{Distortion Effects on Solution Accuracy}

Optical distortion affects AVGS navigation solutions in three principal mechanisms:

1) Sufficient distortion (e.g. coma) of the individual target images can exceed target recognition software limits. The targets are expected to return near-circular images at the sensor, and spot aspect ratio (length/width) is used to discard candidate target spots from processing if the aspect ratio on the focal plane exceeds preset limits.

2) Figure 4 summarizes the distortion of individual target images across the field of view at $30 \mathrm{~m}$. Similar distortion of target centroids is observed when the targets are rotated so that the corner cubes optical axes are pointed away from the sensor.

3) General alignment errors, either in-plane of the focal plane, or in roll about the optical axis, will result in erroneous target pointing and alignment vectors from the navigation sensor.

4) If the target cluster extends over any substantial area on the focal plane, spherical distortion can cause varying displacement of the individual target spots within the image frame. This variable distortion causes errors in the perspective inversion solution, resulting in errors in relative attitude (pitch and yaw) measurements which are larger than guidance constraints permit

\section{Distortion Sources (lens, mirror, and CMOS imager positioning)}

If the imager lens exhibits barrel/pincushion distortion, the relative positions of the target images will be distorted, which will cause errors in calculated relative attitude and errors in estimating the location of the ASTRO vehicle relative to the NEXTSat.

This distortion source deserves some special mention. During AVGS assembly, the manufacturer attempted to verify the lens distortion factor using the sensor focal plane as the test detector. Based on their tests, the manufacturer reported that lens distortion was not detectable. Later testing with accurate truth data supplied by precision ground support equipment revealed distortion values large enough to require software correction. What was the difference? Testing during the manufacturing process used a precision collimated source, and estimated the resultant centroid of that source to within one pixel on the sensor focal plane array. AVGS image processing calculates target centroids to approximately one-tenth of a pixel. Lens distortion which was not detected at the manufacturer's test level was found to create substantial errors in practice. 


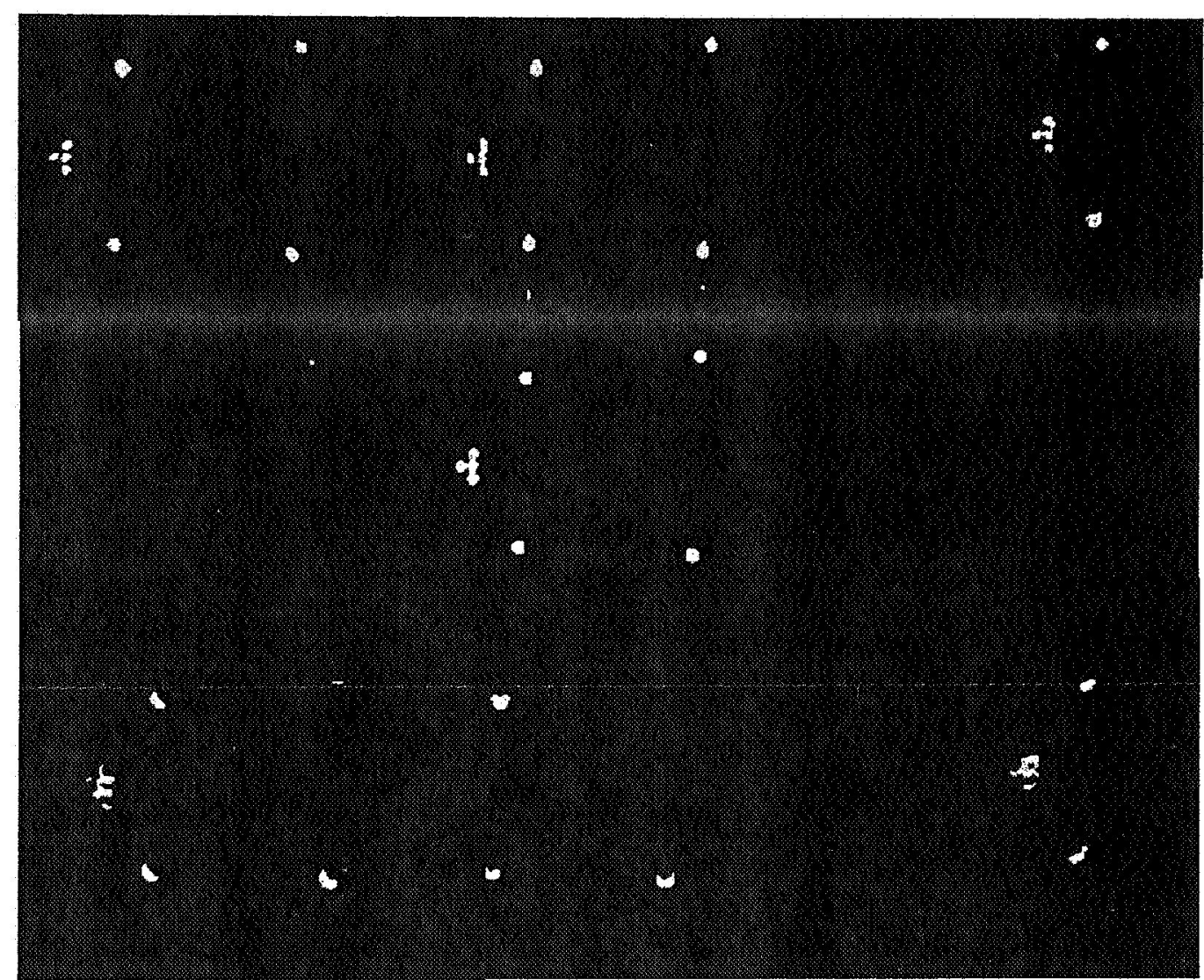

Figure 4. Composite image (not to scale) showing typical image defects at corners of field of view caused by combined focal plane alignment error and corner cube optical behavior

The AVGS images targets through a turning mirror. A hole in the center of the mirror provides access for a fiber feed for the coaxial laser illumination system. Although the coaxial source provides the best possible light return from the corner-cube targets, it also injects an error by eliminating return of photons at the center of the optics. This creates a "hole" in the each target image, as depicted in Figure 5.

The apparent hole in the center of the target moves as the target is rotated in pitch or yaw, which adversely affects the calculated target centroid position. Shifts in the target centroids feed directly into errors in the navigation solution. This error source can become substantial at closer ranges, especially because as the targets spread across the field of view, the centroid shift varies from target to target. At docking ranges the errors induced in target centroid locations were as large as 0.03 degrees before corrections were applied. This is ten percent of the entire error budget for target vector, and could cause relative attitude errors to exceed allowable limits. 


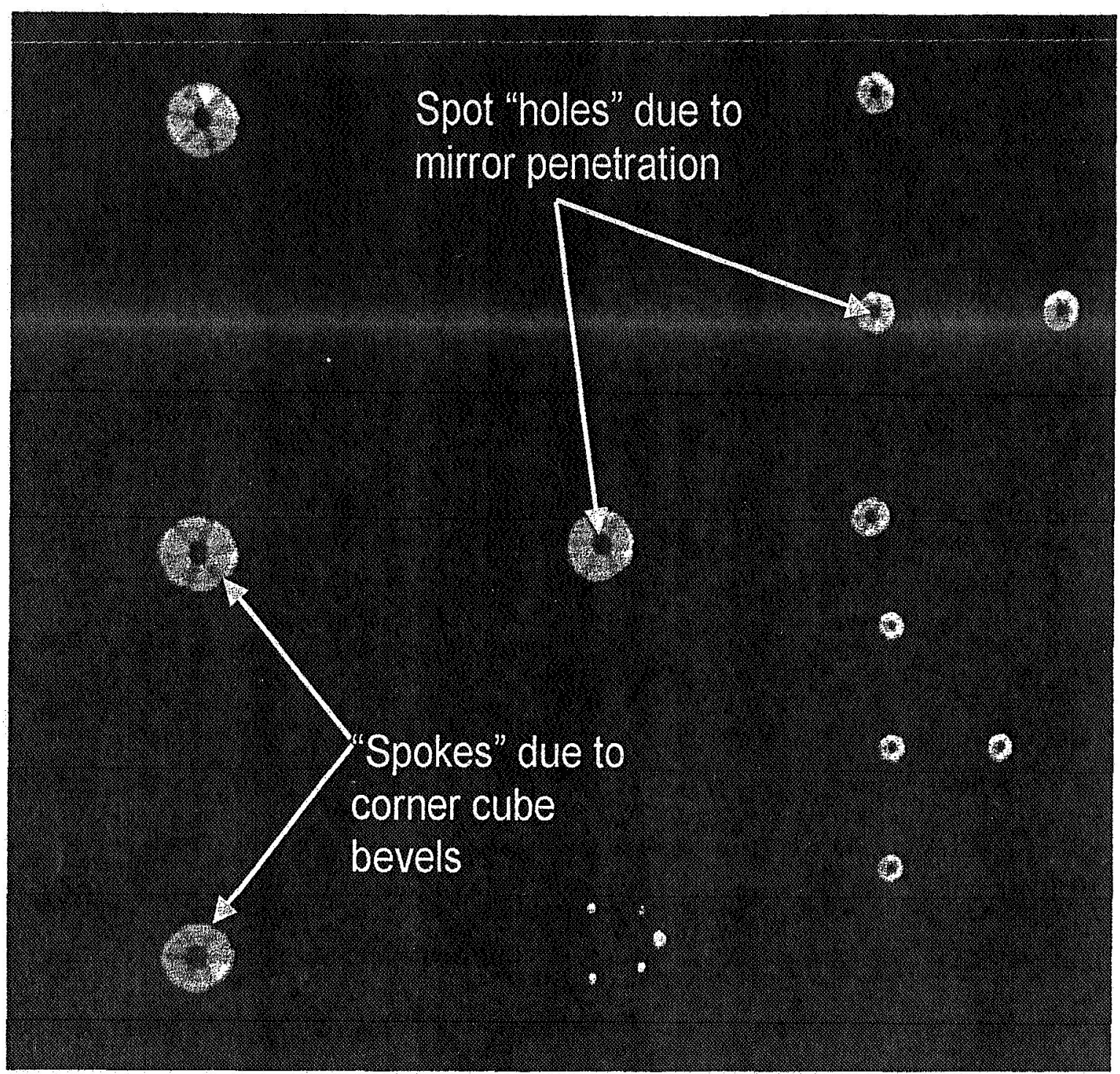

Figure 5. Composite image depicting AVGS corner cube target returns at 1, 2, 3.5, and 15 meters

\section{Magnitude of Distortion/Aberration Defects (and Controls)}

As retro-reflector images deform because the target optical axis is not pointed directly toward the sensor, the image spot centroids move away from the paraxial positions in a predictable manner. Also, as the target retroreflectors move away from the sensor boresight, the spot centroids move in a predictable manner. For AVGSconfiguration retroreflector targets, the target centroids appear to shift approximately $3.3 \%$ of the target aperture diameter per degree of tilt, or 0.06 degrees outboard per degree of sensor tilt at 1.2 meters. This was modeled as an apparent physical shift of the retro-reflector as the sensor tilted. The phenomenon was measured at several ranges and found to be equivalent to a centroid shift of approximately $1.5 \mathrm{~mm}$ per degree of tilt in each axis (azimuth and elevation). Similarly, the corner cube tilt appeared to cause a shift of approximately $0.3 \mathrm{~mm}$ per degree of target tilt. While that is a small amount of shift, at close ranges it caused too much error.

Uncorrected, this shift would cause navigation errors to exceed the sensor error budget. The sensor budget for azimuth and elevation at docking ranges is 0.033 degrees. Errors of 0.06 degrees per degree of tilt cause the sensor error budget to be exceeded very quickly. The errors were corrected in software, and the correction processes verified first in software testing, and then in integrated system tests. 


\subsection{Laser/target optical error sources}

The target illumination system of the AVGS (laser diodes coupled into fiber optics) creates uneven illumination across the FOV. In addition, the lasers have speckle that add noise to target measurements, and the target retroreflectors have variations in their response relative to their apparent size and relative angle.

\section{Illumination/Target Optical Effects on Solution Accuracy}

Uneven illumination of the sensor field of view creates undesirable signal to noise ratios as targets approach the edges of the AVGS field of view. This problem is exacerbated by the requirement to track multiple targets across the FOV. The Gaussian illumination pattern emitted by the laser fibers causes the illumination level in the corners to be much dimmer than the center. This forces lowering of the imager processing threshold. A higher value of threshold is preferred, to eliminate as much background clutter as possible. If the thresholds are set too low, the number of detected spots on the focal plane might be so high that the pattern recognition software would be unable to complete checking for valid target patterns in the time allocated for that task. Lowering thresholds also adds noise to the solution, by admitting lens flare and clutter close to target images into the target centroid calculation. Background noise is quite evident on AVGS images prior to thresholding. Although the imager specification indicates the imager noise floor should be around five to six counts, actual dark-field random noise is observed to be over 30 counts. This higher-than-expected background noise places stringent requirements on exposure parameters, since the sensor needs to produce target images well above the threshold level, without reaching the pixel saturation limits. Saturation of pixels would degrade the accuracy of weighted-centroid calculations, and as a result the overall navigation solution accuracy.

2. Laser Speckle Effects

As ASTRO closes with NEXTSat, the target images become large in the image plane, subtending as many as fifty pixels across at docking range. Speckle from the multi-mode laser sources causes individual pixels in the target images to vary substantially in amplitude. Speckle effects create substantial short-term noise in the navigation solutions. Increased noise due to speckle is mitigated by adding a separate algorithm for centroiding at close range. At longer ranges where speckle is not an issue, the AVGS calculates weighted target centroids. At ten meters, where speckle effects begin to cause solution noise to exceed limits, AVGS software switches to a geometric centroid solution, which is relatively immune to speckle-induced errors.

\section{AVGS Target Geometry Effects}

The AVGS targets are high quality corner cubes, selected for optical efficiency. The AVGS perspective inversion algorithms assume that the centroid of the image formed by light returned from the retroreflector on the sensor focal plane will coincide with the paraxial approximation. This assumption is not valid for cases where the sensor is offaxis from the targets. Retroreflector optics also generates areas of greater and lesser brightness corresponding to the facets and edges of the corner cube glass. If sufficiently severe, the brightness variations within target images could cause target recognition software to perceive a single physical target as multiple independent segments. Typical image behavior is indicated in Figure 6.

. The off-axis effect on solution accuracy primarily affects the relative pitch and yaw components of the navigation solution. As long as the ASTRO vehicle remains in or near the nominal approach corridor, errors due to centroid shift are within allowable limits.

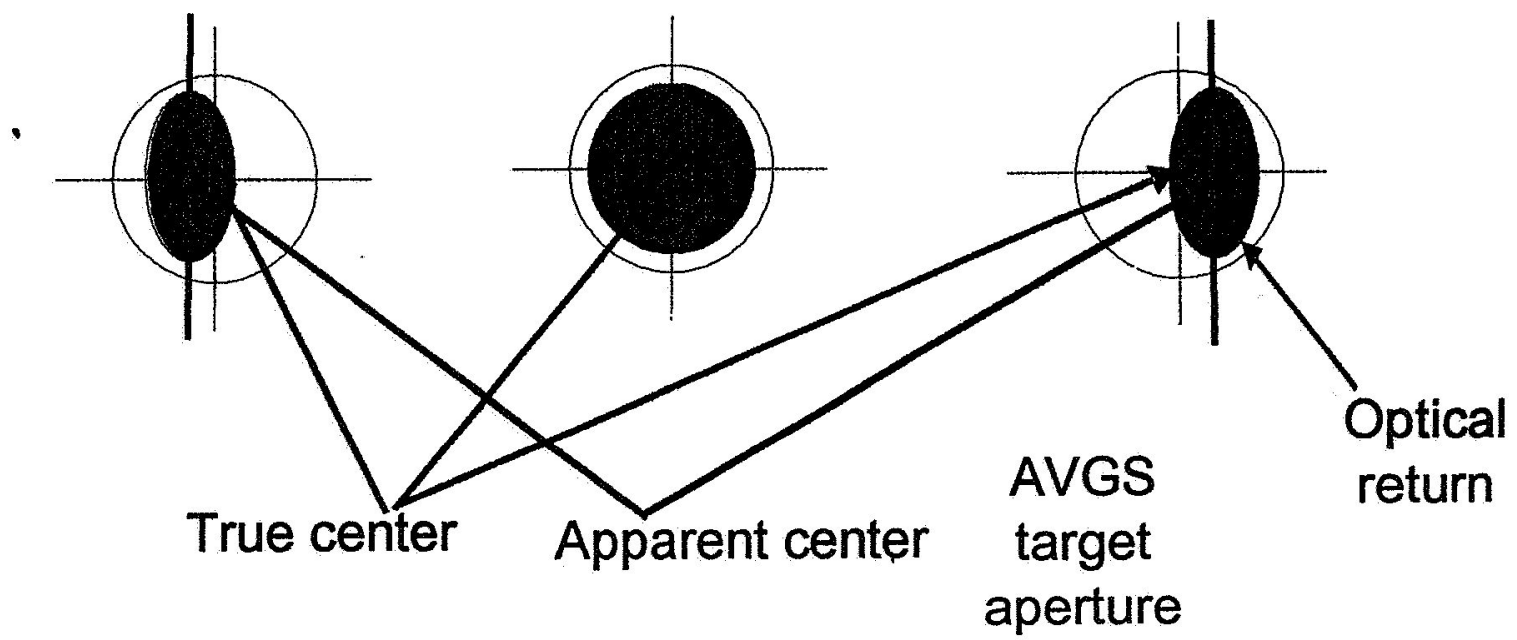

Figure 6: Corner cube centroid displacement due to viewing angle 


\section{MEASUREMENT AND EVALUATION OF OPTICAL DISTORTION EFFECTS ON AVGS SOLUTIONS}

The following algorithm was initially applied to transform the raw XY pixel coordinates into corrected azimuth and elevation angles. This process corrects only for spherical distortion, and does not address errors caused by focal plane position/angular alignment offsets, or by target centroid displacements due to corner cube optics.

1) Correct translational and rotational misalignments:

$$
\left|\begin{array}{l}
X_{M} \\
Y_{M}
\end{array}\right|=\left|\begin{array}{l}
\cos \theta \\
\sin \theta \\
\sin \theta \\
\cos \theta
\end{array}\right|\left|\begin{array}{l}
X-X_{0} \\
Y-Y_{0}
\end{array}\right|
$$

2) Correct optical distortion error (for spherical distortion only):

$$
\begin{gathered}
R_{M}{ }^{2}=X_{M}{ }^{2}+Y_{M}{ }^{2} \\
R_{M}=\sqrt{R_{M}{ }^{2}} \\
R_{C}=K\left(R_{M}\right)
\end{gathered}
$$

3) Compute azimuth and elevation angles:

$$
\begin{aligned}
& A Z=\tan ^{-1}\left(Y_{C} / F A S\right) \\
& E L=\tan ^{-1}\left(X_{C} / F A S\right)
\end{aligned}
$$

where:

FAS (Fixed Adjacent Side) $=\mathrm{K}\left(\mathrm{R}_{8}\right) / \tan 8^{\circ}$

$\mathrm{X}_{\mathrm{M}}=$ Measured $\mathrm{X}$ pixel location after translational and rotational corrections

$\mathrm{Y}_{\mathrm{M}}=$ Measured $\mathrm{Y}$ pixel location after translational and rotational corrections

$\mathrm{X}_{\mathrm{C}}=$ Corrected $\mathrm{X}$ pixel location after translational, rotational and optical distortion corrections

$\mathrm{Y}_{\mathrm{C}}=$ Corrected $\mathrm{Y}$ pixel location after translational, rotational and optical distortion corrections

$\mathrm{R}_{8}=$ Expected pixel location at the 8 degree limit of the field of view (correction limit)

The corrected pixel locations are passed on to the perspective inversion algorithm for calculation of the client vehicle's relative attitudes, range, and bearings. The perspective inversion algorithm is discussed in detail in Ref. 2. 
NASA/MSFC personnel tested AVGS performance exhaustively at ranges between docking and 100 meters, using precision gimbal/translation mounts to obtain accurate truth data. NASA engineers determined that the method of correcting only for spherical-distortion was not adequate given the observed errors in navigation state estimates. Figure 7 depicts the test setup in schematic form.

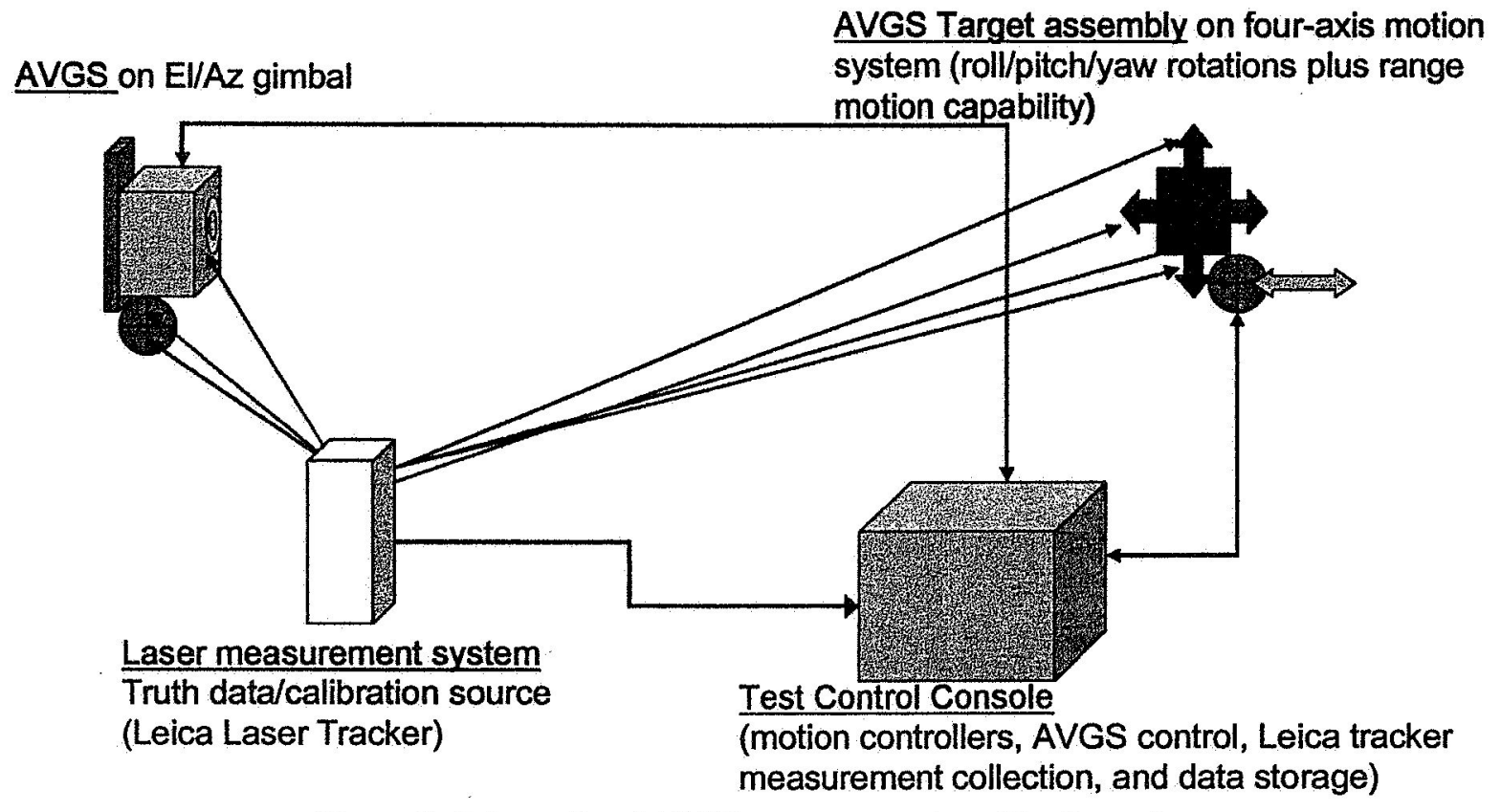

Figure 7: Schematic of AVGS measurement verification setup

The improved algorithms used on the flight AVGS correct for optical distortion in each quadrant of the sensor field of view separately. Application of this second level of correction for optical effects allows the AVGS to meet measurement performance requirements with as-built alignment of the sensor optical train.

\subsection{Details and Effectiveness of Corrections}

Initial AVGS testing was dependent on minimizing the differences between the relative position resulting from the short range target solution and the long range target-derived solution. This approach was reasonable for developing and testing overall sensor functionality and verifying algorithm suitability, however, to provide the precision data required for a docking sensor with the accuracies noted in paragraph 1.3, a method of acquiring more accurate truth data was developed. This data was collected using precision laser trackers to survey the locations of the sensor and the individual target retroreflectors. A series of system accuracy tests were run at these surveyed locations using the precision gimbal/translation mounts. These tests were iterative in that calibration values were determined to yield the desired performance at each selected test range. Ultimately, these tests demonstrated that calibrations could be applied such that the sensor will meet the operational performance requirements. The resulting calibration parameters were captured in as part of the instruments initialization load (or ILOAD) table.

All centroid calculations are applied before the target centroid positions are passed on to the inverse perspective algorithm for calculation of the relative navigation state. The centroid correction process is:

1) The LOS pitch and yaw to each corner cube is computed by the equations

$$
\begin{aligned}
& \text { PitchLOS }=\text { Pitch_Deg }- \text { Elevation_Deg } \\
& \text { YawLOS }=\text { Yaw_Deg }- \text { Azimuth_Deg }
\end{aligned}
$$


Pitch and Yaw derive from the previous Inverse Perspective solution

Azimuth and Elevation derive from raw target position.

2) The initial corrected azimuth and elevation are based on the following equations:

$$
\begin{aligned}
& \text { InitialAz }=\mathrm{Az} /(1+\operatorname{atan}(\mathrm{fCl} / \mathrm{R}))-\text { YawLOS*atan }(\mathrm{ITC} / \mathrm{R}) \\
& \text { InitialEl }=\mathrm{El} /(1+\operatorname{atan}(\mathrm{fC} 1 / \mathrm{R}))-\text { PitchLOS*atan(ITC/R })
\end{aligned}
$$

where $\mathrm{R}$ is the range, ITC is the ILOAD Tilt Constant, and $\mathrm{fC} 1$ is a constant.

3) Final correction is made to azimuth and elevation by subtracting off adjustments based on a third-order equation with coefficients that can be different in each of the four quadrants of the FOV.

$$
\begin{aligned}
\text { FinalAz }= & A z-\operatorname{atan}\left(\mathrm{C} 1 * \text { InitialAz }{ }^{3}+\mathrm{C} 2 * \text { InitialAz }^{2}+\mathrm{C} 3 * \text { InitialAz }\right) \\
& - \text { YawLOS } * \operatorname{atan}(\mathrm{ITC} / \mathrm{R}) \\
\text { FinalEl }= & \mathrm{El}-\operatorname{atan}\left(\mathrm{C} 1 * \text { InitialEl }{ }^{3}+\mathrm{C} 2 * \text { InitialEl }^{2}+\mathrm{C} 3 * \text { InitialEl }\right) \\
& - \text { PitchLOS } * \operatorname{atan}(\mathrm{ITC} / \mathrm{R})
\end{aligned}
$$

where $\mathrm{C} 1, \mathrm{C} 2, \mathrm{C} 3$, and ITC are all empirically derived constants from the ILOAD table.

\section{CONCLUSIONS}

In testing the AVGS and accommodating some of the artifacts its optics insert in the navigation solutions, valuable lessons were obtained applicable to future design of navigation instruments.

Optics design and manufacture need to provide for accurate alignment of the focal plane with imaging optics to minimize pitch and yaw errors in focal plane placement. Distortion caused by the focal plane normal misalignment with the lens optical axis is challenging to compensate for in software. Even after software correction, focal plane angular misalignment will produce detectable bias error. Misshapen images caused by focal plane positioning and angular alignment errors can be difficult to accommodate in target recognition software.

A coaxial light source offers the best photon return efficiency and hence best signal/noise ratio in the system. An unplanned feature of the coaxial illumination system is that the penetration in the AVGS mirror creates dark spots in the center of AVGS target images. These dark spots move from the target image center if the target is rotated in pitch or yaw. The decentering creates errors in the navigation solution. Substantial improvements in the solution stability/accuracy can be obtained by reducing the image effects of the non-reflecting penetration in the AVGS mirror. This could be accomplished by any of several approaches:

1) Reducing the output fiber diameter, and with it, the penetration size

2) Moving the mirror further from the AVGS lens to reduce the subtended angle for the hole

3) Firing the laser through a beam splitter mirror or pellicle, to eliminate the hole altogether

Similarly, the corner cube reflectors used in the Orbital Express targets are extremely efficient at returning laser light to the sensor. The internal reflections in the corner cubes cause apparent motion in the target centroids, displacing target centroids from the desired paraxial location. This error could be compensated for in future systems software, or eliminated by using a microsphere or other similar surface-type retroreflector.

Another unexpected optical artifact was the appearance of corner cube bevels in the sensor raw images. The AVGS targets are built around high-quality solid corner cubes, with $0.25 \mathrm{~mm}$ bevels. The very small bevels were selected to ensure that the target recognition software would be presented with one continuous spot on the image plane for each target. With initial exposure parameters and threshold levels, individual targets could be identified by software as six separate spots. Careful selection of exposure parameters and thresholds brought levels up in the images around bevels to ensure that target recognition software would identify each target as a single spot. 
Although surface-type retroreflectors are lower efficiency than corner cubes, a system designed to accommodate lower photon returns could simplify the target recognition task. Flat-panel targets would also be lighter and considerably less expensive than the precision corner cube assemblies used on the Orbital Express mission. This option was not available on the Orbital Express system, due to the limited sensitivity of the AVGS Photobit focal plane array and power limits on AVGS lasers.

Space navigation applications place a real premium on uniformity of illumination in the instrument field of view. If the AVGS fiber emitter numeric aperture were to be increased from the current flight unit value of 0.22 to 0.35 , the improved illumination uniformity would increase the signal to noise ratio at the edges of the 16 degree field of view by approximately $24 \%$ and at the corners by $43 \%$, compared to illumination at the center of the field of view. Since the AVGS focal plane provides only eight bits per pixel, the improvement in attainable signal to noise ratio is substantial.

Designers of future space sensors using the perspective inversion approach to derive navigation data may obtain improved system performance by taking lessons learned on AVGS development into account. Some tasks that will facilitate development of future systems include:

1) Detailed analysis of the system optics, including lens aberration and alignment error effects

2) Use of special tooling to accurately position the sensor focal plane, without relying on raw imager data to indicate adequate alignment

3) Producing a thorough link budget that considers real-world sensor noise and sensitivity, and the illuminator power and distribution pattern

4) Sophisticated thermal control design to prevent unplanned thermal deflections within the optics train

5) System verification with precision measurement for truth data

6) Analysis of proposed target geometry using perspective inversion to detect potential solution singularities or other computational issues caused by the target pattern

Sensors similar to the AVGS will be key parts of future space missions that rely on vehicular rendezvous and docking for resupply of operational and exploration spacecraft. Building on lessons learned on the AVGS development effort can make those future systems more robust and less costly.

\section{Acknowledgments}

The authors wish to express sincere appreciation to the key managers at NASA, DARPA, and Boeing who stayed the course through a difficult development process that in the end delivered a reliable sensor for the Orbital Express program.

Without the continuing support of Mr. James E. Lee at NASA, Lt. Col. Fred Kennedy at DARPA, and Mr. Rich Matthews at Boeing, this project could not have been completed.

\section{References}

'Granade, S. R., and LeCroy, J., "Analysis and Design of Solid Corner Cube Reflectors for a Space Navigation Application," Proceedings of SPIE - Volume 5798, 2005, pp. 120-129.

${ }^{2}$ Calhoun, P. C., and Dabney, R., "A Solution to the Problem of Determining the Relative 6 DOF State for Spacecraft Automated Rendezvous and Docking", Proceedings of SPIE - Volume 2466, 1995, pp. 175-184. 